\title{
Ketamine acts as an internal contextual stimulus that facilitates the retrieval of weak associations in conditioned taste aversion
}

\author{
PHILIPPE OBERLING \\ Laboratoire de Psychopathologie et de Pharmacologie de la Cognition \\ INSERM, Strasbourg, France \\ GEORGES DI SCALA \\ Laboratoire de Neurosciences Comportementales et Cognitives \\ CNRS, Strasbourg, France \\ and \\ GUY SANDNER \\ Laboratoire de Psychopathologie et de Pharmacologie de la Cognition \\ INSERM, Strasbourg, France
}

\begin{abstract}
Ketamine $(6 \mathrm{mg} / \mathrm{kg})$, when injected intraperitoneally into Long-Evans rats during both the acquisition and the test phases, was found to facilitate the retrieval of a conditioned taste aversion (CTA) based on pairings of a flavor with lithium chloride $(\mathrm{LiCl})$. This facilitation was not a monotonic function of $\mathrm{LiCl}$ dosage, since it was observed with only $15.90 \mathrm{mg} / \mathrm{kg}$ and not $7.95,31.80,63.60$, or $127.20 \mathrm{mg} / \mathrm{kg}$. In our conditions, $15.90 \mathrm{mg} / \mathrm{kg}$ of $\mathrm{LiCl}$ is a dosage that elicits a weak CTA. This result suggests that ketamine acts like a contextual cue, which facilitates the retrieval of weak but not of strong associations. This result is discussed in the framework of drug-induced state dependency, and compared with the effects of external contextual cues.
\end{abstract}

State-dependent learning-or more precisely, statedependent retrieval (SDR)-refers to the fact that information learned in a given state can be recalled better or exclusively when a subject is tested in this state, but not when the subject is tested in a state different from that in which the information was acquired (Overton, 1978; Weingartner, 1978). A variety of states have been shown to elicit SDR, such as affective states (see, e.g., Bower, 1981), endogenous biorhythm-induced states (e.g., Evans, Gustafson, O'Connell, Orne, \& Shor, 1966; Holloway, 1978), or drug-induced states (see Overton, 1982). Druginduced SDR is still considered by some authors as a nonspecific, degenerate phenomenon, closely dependent on the discriminative properties of the pharmacological agents (Overton, 1982; Järbe, 1986, 1987). This view was initially prompted by the observation that the doses of drugs at which state dependency had typically been observed were found to be intoxicating (Overton, 1966, 1982) or sublethal (Overton, 1972), and thus too high to be relevant to the characteristic behavioral actions of these agents. However, Colpaert $(1986,1990)$ and Jack-

This study was supported by Grant 92170 from the Ministère de la Défense Nationale (DRET). We gratefully acknowledge S. Ahmed, R. R. Miller, and N. E. Spear for their helpful comments during the preparation of this manuscript. Requests for reprints should be addressed to P. Oberling, INSERM U405, 11 rue Humann, 67085 Strasbourg Cedex, France (e-mail: oberling@alsace.u-strasbg.fr). son, Koek, and Colpaert (1992), testing benzodiazepines and NMDA antagonists, have recently demonstrated that drug-induced SDR constitutes a robust phenomenon with low doses of drugs that do not disrupt behavior. Consequently, Colpaert proposed that state dependency could constitute a rather general mechanism by which drugs act on memory processes (see also Colpaert, Niemegeers, \& Janssen, 1976).

Among the various possible mechanisms that have been proposed for SDR, one is that a drug could constitute an internal contextual cue that modulates memory processes in the same way as do external contextual cues; that is, the SDR phenomenon could be seen as an instance of contextual learning. To date, this hypothesis has been supported mainly by the general finding that a shift of drugged state between the acquisition and the retrieval phases impairs performances, just as is observed with a shift of external context (for a review, see Overton, 1985; see also Bouton, Kenney, \& Rosengard, 1990).

Additionally, in favor of the hypothesis that druginduced internal cues act as a context, changes in exteroceptive context and in drug state have been found to interact to affect retrieval (see, e.g., Connelly, Connelly, \& Epps, 1973; Connelly, Connelly, \& Nevitt, 1977; Connelly, Connelly, \& Phifer, 1975). For example, Duncan (1979) showed that the SDR effects of ethanol were insignificant if retrieval testing occurred in the same compartment as that in which the animals had been trained, but 
were obvious if the animals were tested in a different compartment. In Duncan's study, the change in external contextual cues apparently acted to unmask an SDR effect that was not expressed without a concurrent environmental contextual change.

External contextual cues have been repeatedly shown to control associations between stimuli, but only to the extent that the strength of an association is low enough to support modulation (e.g., Bonardi, Honey, \& Hall, 1990; Lovibond, Preston, \& Mackintosh, 1984). When the strength of the association increases, the modulation by external contextual cues tends to disappear. To date, whether this characteristic aspect of external contextual control applies to drugs remains to be tested. If a drug that induces SDR acts as a contextual cue, one would expect to observe this effect-that is, a drug-induced modulation for weak but not for strong associations.

The present experiment was designed to test this prediction in a conditioned taste aversion (CTA) paradigm. The CTA paradigm was used because, as a one-trial learning paradigm, it allows the animals to receive only one injection for acquisition and one injection for testing, thereby avoiding problems of tolerance or sensitization that are encountered with repeated injections. CTA is a situation in which a conditioned stimulus CS (a taste) is paired with an unconditioned stimulus US ( $\mathrm{LiCl}$-induced sickness in the present experiment). To vary the strength of the association, the magnitude of the US was modified by changing the dose of $\mathrm{LiCl}$, thereby modulating the induced illness (Nachman \& Ashe, 1973). The drug selected to induce the internal cue was the noncompetitive NMDA antagonist ketamine at a nondebilitating dose $(6 \mathrm{mg} / \mathrm{kg})$. Ketamine was selected because it has been repeatedly shown to act on learning and memory processes in a state-dependent manner in a CTA paradigm (Aguado, San Antonio, Perez, Del Valle, \& Gomez, 1994) as well as in an operant paradigm (Jackson et al., 1992). The typical procedure of state-dependency research was used (viz., a $2 \times 2$ design), with four conditions differing as a function of whether the subjects were conditioned under ketamine or saline and tested under ketamine or saline (Conditions Ket-Ket, Ket--Sal, Sal-Sal, and Sal-Ket). Each condition was then subdivided into five groups, each of which received a dose of $\mathrm{LiCl}$ ranging from 7.95 to $127.20 \mathrm{mg} / \mathrm{kg}$.

\section{METHOD}

\section{Subjects}

One hundred sixty naive male Long-Evans rats (Janvier, France) weighing $280 \pm 30 \mathrm{~g}$ were used. They were housed, 2 per cage, in a colony room maintained on a 12:12-h light:dark cycle (light on at 0800 ) with food and water provided ad lib.

\section{Drugs}

Ketamine (Imalgène, Rhône Mérieux, France) was dissolved in saline. Ketamine $(6 \mathrm{mg} / \mathrm{kg})$ and saline were injected intraperitoneally (i.p.) in a volume of $1 \mathrm{ml} / \mathrm{kg}$ of body weight.

\section{Procedure}

The animals were randomly allocated to one of two samples ( $n \mathrm{~s}=80$ ), with one sample conditioned under ketamine, the other under saline. Each of these two samples was subdivided into five numerically equivalent subsamples $(n s=16)$, according to the volume of $\mathrm{LiCl}(0.15 \mathrm{M})$ that they received: $1.25,2.50,5.00,10.00$, or $20.00 \mathrm{ml} / \mathrm{kg}$ (i.e., $7.95,15.90,31.80,63.60$, or $127.20 \mathrm{mg} / \mathrm{kg}$, respectively). The experiment began following a series of three daily handling sessions and was conducted daily, always at about the same time.

The animals were placed on a water deprivation schedule the day before the study began. The daily drinking sessions throughout the experiment lasted $5 \mathrm{~min}$ and were conducted during the morning period $(0900-1300 \mathrm{~h})$ in a test cage $(30 \times 25 \times 35 \mathrm{~cm})$. The solutions were presented at room temperature. During the evening $(1900 \mathrm{~h})$, the animals were allowed access to tap water in their home cage for $20 \mathrm{~min}$. The procedure lasted 11 days and was divided into four consecutive phases. During the first 7 days, the animals were shaped to drink tap water from a "Richter" tube in the test cage. On Day 8 (the conditioning day), the animals were injected with ketamine or saline 10 min prior to the experimental sessions and were then allowed to drink from this tube a sweet solution of $.06 \% \mathrm{~K}^{+}$acesulfam (Sunett, Hoechst, Germany). Within 5 min after the end of the drinking session, the animals were injected i.p. with $\mathrm{LiCl}$. At the end of the conditioning day, each subsample was subdivided into two groups of 8 animals, with an equal mean amount of $\mathrm{K}^{+}$acesulfam consumed by each group of a given subcondition. Days 9 and 10 were reshaping days, during which the animals had access to tap water. On Day 11 (the test day), each subsample was injected 10 min prior to the test session, one group being injected with ketamine, the other with saline. All the animals were then allowed to drink the sweet solution (one-bottle test).

\section{Data Analyses}

The volume consumed each day by the animals was measured to the nearest $0.5 \mathrm{ml}$. A suppression ratio was computed. For that, the volume consumed during the test day was divided by the sum of the volume consumed during the conditioning day and the test day. Thereafter, a suppression score was obtained by subtracting the suppression ratio from 1 . Thus, a score of 1 indicates a total suppression of consumption during the test; a score of less than 0.5 indicates an increase of consumption during the test day, compared with the conditioning day. Data were analyzed in a two-way analysis of variance (ANOVA), with condition (4 levels) and dose of $\mathrm{LiCl}$ ( 5 levels) as between-subjects factors. The Brown-Forsythe correction was used for the ANOVA, since the variances were not equal. Post hoc analysis was performed using a Bonferroni multiple $t$ test with alpha adjusted for 30 comparisons (i.e., 6 comparisons for each of the 5 doses of LiCl: Sal-Sal vs. Sal-Ket; Sal-Sal vs. Ket-Sal; Sal-Sal vs. Ket-Ket; Sal-Ket vs. Ket-Sal; Sal-Ket vs. Ket-Ket; Ket-Sal vs. Ket-Ket [BMDP; Dixon, 1988]). Additional trend analyses were realized for each condition, by using the method of orthogonal polynomials (Keppel, 1982).

\section{RESULTS}

Figure 1 depicts the mean score of CTA for each group. For each condition (Sal-Sal, Ket-Ket, Sal-Ket, or Ket$\mathrm{Sal}$ ), the suppression score increased with the dose of $\mathrm{LiCl}$. With the exception of the $15.90-\mathrm{mg} / \mathrm{kg}$ dose of $\mathrm{LiCl}$, all the groups exhibited a roughly similar score of CTA for a given dose of $\mathrm{LiCl}$. With the dose of $15.90 \mathrm{mg} / \mathrm{kg}$ of $\mathrm{LiCl}$, Group Ket-Ket displayed a higher score than the scores observed in the other groups injected with the same 


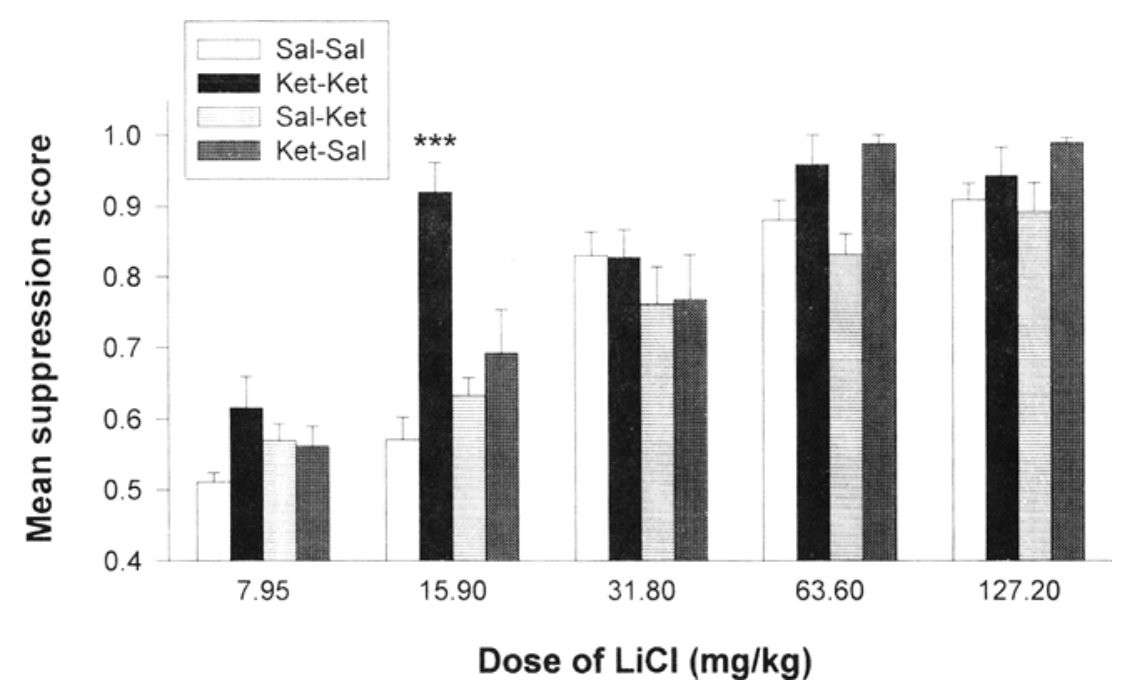

Figure 1. Mean suppression score for the four treatment conditions (SaL-Sal, Ket-Ket, Sal-Ket,
Ket-Sal) according to the dose of $\mathrm{LiCl}$ received by the rats. A score of 1.0 means a complete sup-
pression of drinking during the test day. A score of less than 0.5 indicates an increase of drinking
during the test day, relative to the conditioning day. For each value, $n=8 . * * * p<.001$.

dose. A two-way ANOVA showed a significant effect of condition $[F(3,86)=11.07, p<.0001]$, a significant effect of doses of $\operatorname{LiCl}[F(4,86)=68.50, p<.0001]$, and a significant interaction of condition $\times$ doses $[F(12,86)=$ $3.33, p<.001]$. The post hoc analysis showed that, when the four groups injected with the same dose of $\mathrm{LiCl}$ were compared, Group Ket-Ket injected with $15.90 \mathrm{mg} / \mathrm{kg}$ of $\mathrm{LiCl}$ was the only one that differed from the others $[t(140)=$ $6.67,4.35,5.48$, all $p$ s $<.001$ for Groups Sal-Sal, KetSal, and Sal-Ket, respectively].

In order to further characterize the interaction between condition and doses of $\mathrm{LiCl}$, a trend analysis was separately performed on each of the four conditions. Figure 2 depicts, for each of these conditions, the individual scores of CTA and the relationship between these scores and the doses of $\mathrm{LiCl}$ on a semilogarithmic scale. As can be seen in Figure 2, Condition Sal-Sal exhibited little suppression of consumption at the two lowest doses of $\mathrm{LiCl}$ and strong suppression at the three remaining doses, the transition being located between 15.90 and $31.80 \mathrm{mg} / \mathrm{kg}$. For this condition, the trend analysis detected a sigmoidal relationship between the log of the dose of $\mathrm{LiCl}$ and the effects observed $\left[F_{\text {quart }}(1)=6.96, p_{\text {quart }}<.02\right]$. Contrastingly, Conditions Sal-Ket and Ket-Sal yielded a suppression score which increased with the dose of $\mathrm{LiCl}$ in a linear $\log$ relationship $\left[F_{\operatorname{lin}}(1)=54.06, p_{\text {lin }}<\right.$ $.0001 ; F_{\text {quad }}(1)<1$; and $F_{\text {lin }}(1)=75.91, p_{\text {lin }}<.0001$; $F_{\text {quad }}(1)<1$, for Conditions Sal-Ket and Ket-Sal, respectively]. Finally, Condition Ket--Ket also exhibited a dose-dependent CTA. However, unlike in the other conditions, a quasi-ceiling effect was observed as early as $15.90 \mathrm{mg} / \mathrm{kg}$ of $\mathrm{LiCl}$. Moreover, for this condition, the trend analysis indicated that the suppression score was rather different from that for the other conditions, since a quadratic function of the $\log ($ dose of $\mathrm{LiCl})$ fit the data $\left[F_{\text {quad }}(1)=7.28, p_{\text {quad }}=.01 ; F_{\text {cub }}(1)=3.64\right]$.

\section{DISCUSSION}

The present study shows that when injected both during the acquisition and the retrieval phases, a low dose $(6 \mathrm{mg} / \mathrm{kg})$ of ketamine enhances animals' performances in a CTA paradigm. However, this enhancement is observed only for one out of the five doses of $\mathrm{LiCl}$ used here-namely, $15.90 \mathrm{mg} / \mathrm{kg}$. This enhancement is thought to reflect a ketamine-induced facilitation of memory retrieval rather than any nonmnemonic process, such as an antidipsic or/and a proaversive effects of the drug. The group Ket-Ket injected with $7.95 \mathrm{mg} / \mathrm{kg}$ of $\mathrm{LiCl}$ exhibited the same suppression score as that of the three other groups injected with $7.95 \mathrm{mg} / \mathrm{kg}$ of $\mathrm{LiCl}$, which rules out the possibility that ketamine-induced antidipsia occurred in this study. Ketamine-induced proaversive effects (i.e., ketamine had potentiated LiCl-induced effects) should have been detected equally in Conditions Ket-Sal and Ket-Ket, since all the groups in these two conditions received ketamine during the acquisition.

Our results show that ketamine-induced facilitation is only evidenced for a weak CTA. A dose of $15.90 \mathrm{mg} / \mathrm{kg}$ of $\mathrm{LiCl}$ is a low dosage that is not conventionally used to induce a CTA. Ordinarily, CTA paradigms use higher dosages of $\mathrm{LiCl}$, generally ranging from 31.80 to $127.20 \mathrm{mg} / \mathrm{kg}$ (see, e.g., Archer, Sjödén, \& Nilsson, 1985; Hall \& Channell, 1986). However, $15.90 \mathrm{mg} / \mathrm{kg}$ of $\mathrm{LiCl}$, as well as $7.95 \mathrm{mg} / \mathrm{kg}$, induced CTAs-but weak ones, as is attested in Condition Sal--Sal by moderate suppression scores between .5 and .6 . When they were injected with saline rather than $\mathrm{LiCl}$, our animals exhibited an increase 


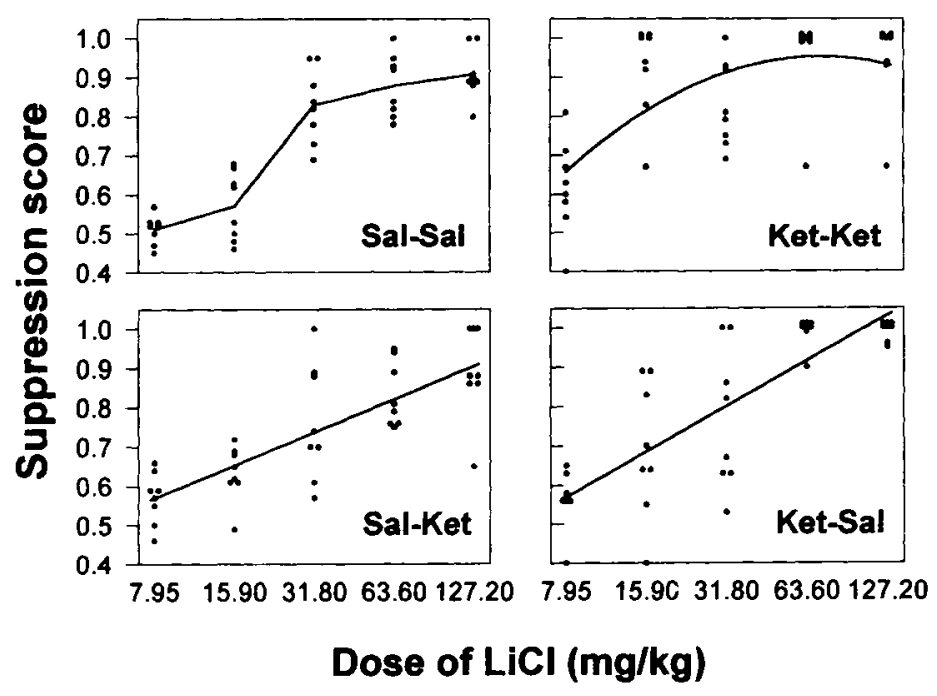

Figure 2. Individual suppression score for the four treatment conditions (Sa-Sal, Ket-Ket, Sa-Ket, Ket-Sal) according to the dose of LiCl received by the rats. Data are presented on a semilogarithmic scale. Each dot represents the score for one rat. For each group, the solid line represents the dose relationship as determined by the trend analysis (see text for details).

of consumption during testing compared with the acquisition, with a typical score of .4. This is thought to reflect the initial neophobic response of rats against $\mathrm{K}^{+}$acesulfam, which is seen with most novel tastes (see, e.g., Barnett, 1958; Domjan, 1977).

Contrastingly, we did not observe ketamine-induced facilitation with strong CTA. Indeed, no significant difference was observed between Groups Sal-Sal and KetKet injected with $31.80 \mathrm{mg} / \mathrm{kg}$ of $\mathrm{LiCl}$; both groups displayed high suppression scores. This absence of difference cannot be attributed to a ceiling effect, because a complete suppression of drinking was observed in only 1 of the 8 rats in Group Ket-Ket. The latter result argues against the fact that ketamine-induced facilitation observed with $15.80 \mathrm{mg} / \mathrm{kg}$ of $\mathrm{LiCl}$ could result from the potentiation by the taste of an aversion to ketamine, as has been reported with, for example, taste-odor combinations (see, e.g., Rusiniak, Hankins, Garcia, \& Brett, 1979).

The trend analysis showed that the results displayed by the five Ket-Ket groups could be described with a quadratic function. This suggests that the facilitation observed at $15.90 \mathrm{mg} / \mathrm{kg}$ of $\mathrm{LiCl}$ is not erratic, but rather belongs to a continuum of effects. When one considers Condition Sal-Sal, the transition between a weak and a strong CTA is located just beyond $15.90 \mathrm{mg} / \mathrm{kg}$ of $\mathrm{LiCl}$. This suggests that ketamine-induced facilitation is, in fact, a shift toward the lowest doses of $\mathrm{LiCl}$ in comparison with what is observed in a traditional CTA - that is, in the condition Sal-Sal-as if, in the presence of a stable cue (viz., ketamine), the animals had a more sensitive representation of the US or of the CS-US association. This is precisely how external contextual cues are believed to act during retrieval. As a matter of fact, Konorski
(1967), Medin (1975), and Spear (1973) have suggested that the external training context "facilitates" retrieval of association between the nominal CS and US; that is, the presence of the external conditioning context during testing enhances the effectiveness of the CS in eliciting conditioned responding. More precisely, Medin (1975) and Spear (1973) hypothesize that the context serves as a cue for the retrieval of associations to the $\mathrm{CS}$ as the result of its being an attribute of the target memory system (see also Miller \& Matzel, 1988; Miller \& Schachtman, 1985). Nadel and Willner (1980) advocate a similar view-specifically, that a hierarchical relationship of cues exists in which contexts both contain and predict CSs.

To summarize, the present study suggests that ketamineinduced facilitation parallels the facilitation observed with external contextual cues. Indeed, ketamine is used by subjects to index learning, and such indexing facilitates retrieval under the following conditions. First, facilitation is observed when the strength of the association between stimuli is low; second, ketamine must be present during both the acquisition and the test phases to be efficient.

Besides facilitation, in the present study we did not observe ketamine-induced SDR. Whenever a shift of state (Sal-Ket or Ket-Sal) between the acquisition and the retrieval phases occurred, we observed a suppression score similar to the one observed in Condition Sal-Sal. A dose of $6 \mathrm{mg} / \mathrm{kg}$ of ketamine is probably too low to allow us to observe SDR in a CTA paradigm. Indeed, on the one hand, Jackson et al. (1992) found evidence for SDR with a similar dose range, but with animals experiencing several days of training under ketamine. On the other hand, Aguado et al. (1994) found evidence for SDR in a one- 
trial CTA paradigm, but with a high, debilitating dose of $25 \mathrm{mg} / \mathrm{kg}$ of ketamine. This suggests that a one-trial predisposed type of learning such as CTA is probably poorly sensitive to changes of state and therefore requires massive dosages to exhibit clear drug-induced SDR. Nevertheless, in our conditions, $6 \mathrm{mg} / \mathrm{kg}$ of ketamine may have impaired memory processes in an SDR manner, but this impairment may have been too weak to affect our results significantly. As a matter of fact, the trend analyses detect a qualitative differential $\mathrm{LiCl}$-induced dose relationship for Condition Sal-Sal (a sigmoidal one) and for Conditions Sal-Ket and Ket-Sal (linear ones). When one considers the individual suppression scores, it appears that the linear relationships observed were due to the wide dispersion of the performances at $31.80 \mathrm{mg} / \mathrm{kg}$ of $\mathrm{LiCl}$ for Groups Ket-Sal and Sal-Ket, with half of the animals in each of this two groups exhibiting lower suppression scores than the lowest score observed in Group Sal-Sal. This qualitative difference leads us to propose that, at least with ketamine, facilitation and SDR might reflect a continuum of similar memory processes, with drug-induced facilitation being observed with lower dosage than that for drug-induced SDR. In the former case, the drug would be used as a discrete cue, facilitating the retrieval of the target information that has been weakly processed. In the latter case, because of the higher dosage, the drug would lock the target information in the drug state, even when it was more deeply processed, requiring the subject to experience this state to retrieve it. Increasing the processing for this information (by overtraining, for example; see, e.g., Iwahara \& Noguchi, 1972, 1974) will unlock the memory trace from the drug state, allowing the subject to retrieve the information, whatever the present state.

In conclusion, the present results are consistent with the notion that drug-induced SDR is a contextual phenomenon. However, they stress that drug-induced contextual effects should vary according to two distinctive dimensions: a pharmacological one-that is, the dosage of the drug used - and a psychological one - that is, the amount of processing for the target information. This could be an important difference for the way in which drug stimuli and exteroceptive sensory stimuli control learning or retrieval processes. In particular, this could account for the fact that, unlike exteroceptive stimulus control, SDR is so inconstantly observed from one study to another (see, e.g., Aguado et al., 1994, vs. Welzl, Alessandri, \& Bättig, 1990).

\section{REFERENCES}

Aguado, L., San Antonio, A., Perez, L., Del Valle, R., \& Gomez, J. (1994). Effects of the NMDA receptor antagonist ketamine on flavor memory: Conditioned aversion, latent inhibition and habituation of neophobia. Behavioral \& Neural Biology, 61, 271-281.

ARChER, T., SJöDÉN, P. O., \& NilsSon, L.-G. (1985). Contextual control of taste-aversion conditioning and extinction. In P. D. Balsam \& A. Tomie (Eds.), Context and learning (pp. 225-271). Hillsdale, NJ: Erlbaum.

BARNETT, S. A. (1958). Experiments on "neophobia" in wild and laboratory rats. British Journal of Psychology, 49, 195-201.
Bonardi, C., Honey, R. C., \& Hall, G. (1990). Context specificity of conditioning in flavor-aversion learning: Extinction and blocking tests. Animal Learning \& Behavior, 18, 229-237.

Bouton, M. E., Kenney, F. A., \& Rosengard, C. (1990). Statedependent fear extinction with two benzodiazepine tranquilizers. $\mathrm{Be}$ havioral Neuroscience, 104, 44-55

Bower, G. H. (1981). Mood and memory. American Psychologist, 36, 129-148.

COLPAERT, F. C. (1986). A method for quantifying state-dependency with chlordiazepoxide in rats. Psychopharmacology, 90, 144-146.

COLPAERT, F. C. (1990). A mnesic trace locked into the benzodiazepine state of memory. Psychopharmacology, 102, 28-36.

Colpaert, F. C., Niemegeers, C. J. E., \& Janssen, P. A. J. (1976). Theoretical and methodological considerations on drug discrimination learning. Psychopharmacologia, 46, 169-177.

Connelly, J. F., Connelly, J. M., \& EpPs, J. O. (1973). Disruption of dissociated learning in a discrimination paradigm by emotionally important stimuli. Psychopharmacologia, 30, 275-282.

Connelly, J. F., Connelly, J. M., \& Nevitt, J. R. (1977). Effect of foot-shock intensity on amount of memory retrieval in rats by emotionally important stimuli in a drug-dependent learning escape design. Psychopharmacology, 51, 153-157.

Connelly, J. F., ConNelly, J. M., \& Phifer, R. (1975). Disruption of state-dependent learning (memory retrieval) by emotionally important stimuli. Psychopharmacologia, 41, 139-143.

Dixon, W. J. (1988). BMDP statistical sofiware manual. Berkeley: University of California Press.

Domjan, M. A. (1977). Attenuation and enhancement of neophobia for edible substances. In M. L. Barker, M. R. Best, \& M. A. Domjan (Eds.), Learning mechanisms in food selection (pp. 151-180). Waco, TX: Baylor University Press.

Duncan, P. M. (1979). The effect of external stimulus change on ethanol-produced dissociation. Pharmacology, Biochemistry \& Behavior, 11, 377-381.

Evans, F. J., Gustafson, L. A., O'Connell, D. L., Orne, M. T., \& SHOR, R. E. (1966). Response during sleep with intervening waking amnesia. Science, 152, 666-667.

Hall, G., \& Channell, S. (1986). Context specificity of latent inhibition in taste aversion learning. Quarterly Journal of Experimental Psychology, 38B, 121-139.

Holloway, F. A. (1978). State dependent retrieval based on time of day. In B. T. Ho, D. W. Richards, \& D. L. Chute (Eds.), Drug discrimination and state dependent learning (pp. 319-343). New York: Academic Press.

Iwahara, S., \& Noguchi, S. (1972). Drug-state dependency as a function of overtraining in rats. Japanese Psychological Research, 14 141-144.

IWAHARA, S., \& NoGUCHI, S. (1974). Effect of overtraining upon drugstate dependency in discrimination learning in white rats. Japanese Psychological Research, 16, 59-64.

JACKSON, A., KoEk, W., \& ColPAerT, F. C. (1992). NMDA antagonists make learning and recall state-dependent. Behavioural Pharmacology, 3, 415-421.

JäRBE, T. (1986). State dependent learning and drug discriminative control of behavior: An overview. Acta Neurologica Scandinavica, 74, 37-59.

JäRBE, T. (1987). Drug discrimination learning. In A. J. Greenshaw \& C. T. Dourish (Eds.), Experimental psychopharmacology (pp. 433479). Clifton, NJ: Humana Press.

KePpel, G. (1982). Analysis of trend. In G. Keppel (Ed.), Design \& analysis: A researcher's handbook (2nd ed., pp. 127-143). Englewood Cliffs, NJ: Prentice-Hall.

KONORSKI, J. (1967). Integrative activity of the brain: An interdisciplinary approach. Chicago: University of Chicago Press.

Lovibond, P. F., Preston, G. C., \& Mackintosh, N. J. (1984). Context specificity of conditioning, extinction, and latent inhibition. Journal of Experimental Psychology: Animal Behavior Processes, 10, 360375.

MEDIN, D. L. (1975). A theory of context in discrimination learning. In G. H. Bower (Ed.), The psychology of learning and motivation (Vol. 9, pp. 263-314). New York: Academic Press.

MilleR, R. R., \& MATzel, L. D. (1988). The comparator hypothesis: 
A response rule for the expression of associations. In G. H. Bower (Ed.), The psychology of learning and motivation (Vol. 22, pp. 5192). San Diego, CA: Academic Press.

Miller, R. R., \& Schachtman, T. R. (1985). The several roles of context at the time of retrieval. In P. D. Balsam \& A. Tomie (Eds.), Context and learning (pp. 167-194). Hillsdale, NJ: Erlbaum.

NACHMAN, M., \& AsHE, J. H. (1973). Learned taste aversions in rats as a function of dosage, concentration, and route of administration of LiCl. Physiology \& Behavior, 10, 73-78.

NAdEL, L., \& WillNer, J. (1980). Context and conditioning: A place for space. Physiological Psychology, 8, 218-228.

Overton, D. A. (1966). State-dependent learning produced by depressant and atropine-like drugs. Psychopharmacologia, 10, 6-31.

OVERTON, D. A. (1972). State-dependent learning produced by alcohol and its relevance to alcoholism. In B. Kissen \& H. Begleiter (Eds.), The biology of alcoholism: Vol. II. Physiology and behavior (pp. 193217). New York: Plenum.

OVERTON, D. A. (1978). Major theories of state dependent learning. In B. T. Ho, D. W. Richards, \& D. L. Chute (Eds.), Drug discrimination and state dependent learning (pp. 283-318). New York: Academic Press.
Overton, D. A. (1982). Comparison of the degree of discriminability of various drugs using the T-maze drug discrimination paradigm. Psychopharmacology, 76, 385-395.

OverToN, D. A. (1985). Contextual stimulus effects of drugs and internal states. In P. D. Balsam \& A. Tomie (Eds.), Context and learning (pp. 357-384). Hillsdale, NJ: Erlbaum.

Rusiniak, K. W., Hankins, W. G., Garcia, J., \& Brett, L. P. (1979). Flavor-illness aversions: Potentiation of odor by taste in rats. $B e$ havioral \& Neural Biology, 25, 1-17.

SPEAR, N. E. (1973). Retrieval of memory in animals. Psychological Review, 80, 163-194.

WEINGARTNER, H. (1978). Human state dependent learning. In B. T. Ho, D. W. Richards, \& D. L. Chute (Eds.), Drug discrimination and state dependent learning (pp. 361-382). New York: Academic Press.

Welzl, H., Alessandri, B., \& Bättig, K. (1990). The formation of a new gustatory memory trace in rats is prevented by the noncompetitive NMDA antagonist ketamine. Psychobiology, 18, 43-47.

(Manuscript received June 19, 1995; revision accepted for publication July 29,1996 .) 